\title{
Evaluation of mannitol salt agar, CHROMagar Staph aureus and CHROMagar MRSA for detection of meticillin-resistant Staphylococcus aureus from nasal swab specimens
}

\author{
Zhuolin Han, ${ }^{1}$ Ebbing Lautenbach, ${ }^{1,2,3}$ Neil Fishman ${ }^{1,2}$ \\ and Irving Nachamkin ${ }^{4}$
}

Correspondence

Irving Nachamkin

nachamki@mail.med.upenn.edu

Received 12 June 2006

Accepted 10 September 2006

\author{
${ }^{1}$ Department of Hospital Epidemiology and Infection Control, University of Pennsylvania School \\ of Medicine, Philadelphia, PA, USA \\ ${ }^{2}$ Department of Medicine, Division of Infectious Diseases, University of Pennsylvania School of \\ Medicine, Philadelphia, PA, USA \\ ${ }^{3}$ Department of Biostatistics and Epidemiology, Center for Clinical Epidemiology and \\ Biostatistics, University of Pennsylvania School of Medicine, Philadelphia, PA, USA \\ ${ }^{4}$ Department of Pathology and Laboratory Medicine, University of Pennsylvania School of \\ Medicine, Philadelphia, PA, USA
}

\begin{abstract}
Mannitol salt agar (MSA), CHROMagar Staph aureus (CSA) and CHROMagar MRSA (CSAMRSA) were evaluated with nasal surveillance specimens for their ability to detect Staphylococcus aureus and meticillin-resistant $S$. aureus (MRSA). CSA was found to be more sensitive than MSA in detecting $S$. aureus (98 versus $84.3 \% ; P=0.03$ ). CSA and CSA-MRSA were equivalent in the ability to detect MRSA at $24 \mathrm{~h}$ (89.7 versus $87.2 \%$ ) and at $48 \mathrm{~h}$ ( 94.9 versus $94.9 \%$ ). When combined with Staphaurex slide confirmation testing, both CSA and CSA-MRSA were highly specific (100\%) media for detecting MRSA from nasal swab specimens.
\end{abstract}

\section{INTRODUCTION}

Staphylococcus aureus is a major pathogen responsible for nosocomial and community-acquired infection. Meticillinresistant S. aureus (MRSA) has emerged as a nosocomial pathogen of major worldwide importance and is an increasingly frequent cause of community-acquired infections that cause significant morbidity and mortality (Rubin et al., 1999). Colonized personnel can serve as a reservoir for the nosocomial spread of MRSA. Active surveillance and timely identification of MRSA colonization of patients is an important infection control activity that helps to prevent nosocomial spread and is cost-effective (Muto et al., 2003; Wernitz et al., 2005). Our goal was to compare the recovery of MRSA on CHROMagar MRSA with that on other conventional (mannitol salt agar) and chromogenic media (CHROMagar Staph aureus).

\section{METHODS}

From October 2004 to May 2005, we evaluated several culture media for their ability to detect MRSA colonization from surveillance nasal

Abbreviations: CNS, coagulase-negative staphylococci; CSA, CHROMagar Staph aureus; CSA-MRSA, CHROMagar MRSA; MRSA, meticillin-resistant $S$. aureus; MSA, mannitol salt agar. specimens, including mannitol salt agar (MSA), CHROMagar Staph aureus (CSA) and CHROMagar MRSA (CSA-MRSA) (all from BD Diagnostics). Nasal swabs (BBL CultureSwab Liquid Stuart, Double Swab, BD Diagnostics) were prospectively collected from 656 inpatients at the Hospital of the University of Pennsylvania. CSA was initially compared to MSA medium in phase I of the study, prior to the availability of CSA-MRSA from the manufacturer. CSA is a differential medium for the rapid identification of $S$. aureus. Colonies suggestive of $S$. aureus (mauve phenotype) must be further tested for meticillin resistance to confirm the presence of MRSA. For phase I, 316 nasal swab specimens were collected prospectively from patients in the medical intensive care and transplant units, and were sampled upon admission to the unit and weekly thereafter until positive, and used to compare MSA and CSA.

In phase II of the study, 340 nasal swab specimens were collected prospectively from patients in the medical and surgical intensive care units upon admission to the unit, and weekly thereafter until positive, and were used to compare CSA (with cefoxitin disk test confirmation) and CSA-MRSA. Each double swab was emulsified in $2 \mathrm{ml}$ trypticase soy broth, and an equal number of drops were added to CSA, CSAMRSA or MSA. The plates were streaked for isolation and incubated at $37^{\circ} \mathrm{C}$ without $\mathrm{CO}_{2}$ for $24-48 \mathrm{~h}$.

After 18-24 h incubation, mauve colonies on CSA as well as CSAMRSA, and yellow colonies on MSA, were regarded as presumptive $S$. aureus isolates and were subcultured onto $5 \%$ sheep blood agar. All negative cultures at $24 \mathrm{~h}$ were reincubated for an additional $24 \mathrm{~h}$. S. aureus isolates were identified using the catalase test (Humco), Staphaurex slide test (Remel Europe), tube coagulase test (BD 
Table 1. Comparison of MSA and CSA for detection of $S$. aureus $(n=51)$ from nasal swab specimens

\begin{tabular}{|lccccc|}
\hline \multirow{2}{*}{ Medium } & Incubation time $(\mathbf{h})$ & Sensitivity (\%) $(\mathbf{9 5} \% \mathbf{C I})$ & Specificity $(\%) \dagger(\mathbf{9 5} \% \mathbf{C I})$ & Specificity $(\%) \ddagger(\mathbf{9 5} \% \mathbf{C I})$ & $\boldsymbol{P}$ value \\
\hline CSA & 24 & $90.2(78.5,96.7)$ & $99.3(97.4,99.9)$ & $99.2(97.4,99.9)$ & $0.11 \S$ \\
& 48 & $98.0(89.5,99.9)$ & $89.4(85.1,92.9)$ & $99.6(97.9,99.9)$ & $0.03 \|$ \\
MSA & 24 & $76.5(62.5,87.2)$ & $99.6(97.9,99.9)$ & $100(97.8,100)$ & - \\
& 48 & $84.3(71.4,92.9)$ & $95.8(92.7,97.9)$ & $100(97.8,100)$ \\
\hline
\end{tabular}

${ }^{\star} \mathrm{CI}, 95 \%$ confidence interval.

†Without Staphaurex slide test confirmation.

¥With Staphaurex slide test confirmation.

$\S C S A$ versus MSA at $24 \mathrm{~h}$.

IICSA versus MSA at $48 \mathrm{~h}$.

Diagnostics) and Vitek 2 (bioMérieux). Confirmed S. aureus strains were tested using the cefoxitin disk test according to Clinical and Laboratory Standards Institute (2005) guidelines. Coagulase-negative staphylococci (CNS) were identified using the Vitek 2 ID-GP card.

\section{RESULTS AND DISCUSSION}

Among the 316 nasal surveillance specimens that were used to compare CSA and MSA, S. aureus was isolated from 51 samples, of which 19 (37.3\%) were MRSA (Table 1). CSA showed higher sensitivity than MSA at $24 \mathrm{~h}$ (90.2 versus $76.5 \%$ at $24 \mathrm{~h} ; P=0.11$ ), although the difference was not statistically different; CSA was significantly more sensitive at $48 \mathrm{~h}$ ( 98.0 versus $84.3 \%$ at $48 \mathrm{~h} ; P=0.03$ ). For CSA and MSA, it was possible to increase the sensitivity by approximately $8 \%$ by incubating negative plates for an additional $24 \mathrm{~h}$. The specificities of CSA and MSA were 99.3 and $99.6 \%$ at $24 \mathrm{~h}$ and 89.4 and $95.8 \%$ at 48 h, respectively, without the use of Staphaurex slide test confirmation. The specificities improved, however, to 99.2 and $100 \%$ for CSA and MSA, respectively, at $24 \mathrm{~h}$ and 99.6 and $100 \%$ at $48 \mathrm{~h}$, respectively, when the Staphaurex slide test confirmation was used.

Of the 340 nasal surveillance specimens plated on CSA and CSA-MRSA in the phase II analysis, S. aureus MRSA was isolated from 39 samples (Table 2). CSA recovered 89.7 and $94.9 \%$ MRSA at 24 and $48 \mathrm{~h}$, respectively. CSA-MRSA recovered 87.2 and $94.9 \%$ of the MRSA isolates at 24 and $48 \mathrm{~h}$, respectively (CSA versus CSA-MRSA; $P$ not significant). The specificity of both media combined with the Staphaurex slide test was $100 \%$. One MRSA strain was missed by CSA plus cefoxitin disk-diffusion testing but picked up by CSA-MRSA. This was due to a culture containing both MRSA and meticillin-susceptible S. aureus (MSSA), and only the MSSA was picked from the CSA medium for cefoxitin disk-diffusion testing.

False positives which occurred at $24 \mathrm{~h}$ on CSA/CSA-MRSA were all CNS $(n=2)$. False positives at $48 \mathrm{~h}$ on CSA/CSAMRSA were mostly CNS, Staphylococcus epidermidis $(n=5)$, Staphylococcus hominis $(n=1)$, Staphylococcus intermedius $(n=1)$, seven unidentified, and Corynebacterium spp. $(n=2)$. The false-positive yellow colonies on MSA were Bacillus spp. $(n=2)$ and CNS $(n=9)$.

Growth from CSA can be used directly for Staphaurex slide test and susceptibility testing according to the manufacturer's instructions. Performing the Staphaurex slide test directly from mauve colonies increased the specificity of CSA from 99.2 to $99.6 \%$ at $24 \mathrm{~h}$ and from 89.4 to $99.2 \%$ at $48 \mathrm{~h}$.

We also tested 32 random MRSA and 32 CNS from frozen storage. All MRSA and CNS isolates gave the expected phenotypes on both CSA and CSA-MRSA at $24 \mathrm{~h}$

Table 2. Comparison of CSA and CSA-MRSA for detection of MRSA $(n=39)$ from nasal swab specimens

\begin{tabular}{|c|c|c|c|c|c|}
\hline Medium & Incubation time $(\mathbf{h})$ & Sensitivity (\%) $\left(95 \% \mathrm{CI}^{\star}\right)$ & Specificity $(\%) \dagger(95 \%$ CI $)$ & Specificity $(\%) \ddagger(95 \%$ CI $)$ & $P$ value \\
\hline \multirow[t]{2}{*}{ CSA } & 24 & $89.7(75.7,97.1)$ & Not done & $100(97.8,100)$ & $0.7 \S$ \\
\hline & 48 & $94.9(82.7,99.4)$ & & $100(97.8,100)$ & 1.011 \\
\hline CSA-MRSA & 48 & $94.9(82.7,99.4)$ & $94.3(91.1,96.7)$ & $100(97.8,100)$ & - \\
\hline
\end{tabular}

${ }^{\star} \mathrm{CI}, 95 \%$ confidence interval.

$\dagger$ Without Staphaurex slide confirmation test.

\$With Staphaurex slide confirmation test.

§CSA versus CSA-MRSA at $24 \mathrm{~h}, P=0.7$.

IICSA versus CSA-MRSA at $48 \mathrm{~h}, P=1.0$. 
(sensitivity and specificity, $100 \%$ ); this was consistent with the result reported by Diederen et al. (2005), which demonstrated that CSA-MRSA was $100 \%$ sensitive in detecting frozen strains of MRSA. Gaillot et al. (2000) plated 100 frozen strains of $S$. aureus and 45 CNS on CSA and found that all strains showed the desired colony morphology at $24 \mathrm{~h}$. However, the sensitivity and specificity of CSA were 95.5 and $99.4 \%$, respectively, when clinical specimens were plated, and our data were consistent with that study.

Isolates that exhibited false-positive reactions in the phase I and phase II studies on CSA and/or CSA-MRSA were frozen and subsequently retested. Nineteen CNS [S. epidermidis $(n=11)$, S. hominis $(n=4)$, Staphylococcus caprae $(n=1)$, not identified $(n=3)]$ and five Corynebacterium spp. isolates that exhibited false-positive reactions on CSA and/or CSAMRSA were retrieved and replated to CSA and CSA-MRSA. Five of 24 isolates exhibited mauve colonies (one isolate of Corynebacterium spp. at $24 \mathrm{~h}$ on both CSA and CSA-MRSA, three isolates of Corynebacterium spp. and one isolate of $S$. caprae at $48 \mathrm{~h}$ on CSA). This suggests that the bacterial factors that contribute to false-positive reactions on CSA and/or CSA-MRSA are unstable.

CSA-MRSA was found to be comparable with CSA plus the cefoxitin disk-diffusion test in detecting MRSA. It has high sensitivity and specificity when combined with the Staphaurex slide test and allows reporting of MRSA within 1 day. Additionally, CSA-MRSA requires less hands-on time than CSA combined with disk confirmation. Further, using CSA-MRSA medium offers additional time savings ( $\sim 1$ day) compared with CSA combined with cefoxitin disk testing. One limitation of the current study is that we did not confirm all MRSA with testing directly for mecA using molecular methods. The cefoxitin disk test, however, has been shown to be highly sensitive and specific for $m e c A$, and to have an excellent correlation with mecA testing for the detection of MRSA (Swenson et al., 2005).

The results from our study are consistent with the findings from a multicentre study using CHROMagar MRSA medium for detecting MRSA in nasal swab specimens (Flayhart et al., 2005). In that study, CHROMagar MRSA medium had $95.2 \%$ sensitivity and $>96.7 \%$ specificity. CNS and Corynebacterium spp. also occasionally exhibited false positives, as we noted in our survey. Similarly, Stoakes and colleagues compared CHROMagar MRSA medium with other selective media, and for nasal swab specimens, found a sensitivity of $95 \%$ and a high specificity, but did not comment on false positives with this medium (Stoakes et al., 2006). Other chromogenic media, such as S. aureus ID (bioMérieux) (Perry et al., 2003) are also available for detecting S. aureus. S. aureus ID supplemented with cefoxitin (MRSA ID) has been developed by the same manufacturer, and shows comparable sensitivity and specificity to those of CSA-MRSA (Perry et al., 2004).

Several groups have used molecular methods for the rapid detection of MRSA from clinical specimens within a few hours (Francois et al., 2003; Warren et al., 2004). Although molecular methods provide same-day results, they have certain disadvantages, including the need to batch clinical specimens, greater technical demands than culture, expensive reagents, and the need for specialized laboratory equipment.

In summary, our results indicate that CSA-MRSA, or CSA plus the cefoxitin disk diffusion test, are highly effective for detecting MRSA from nasal swab specimens, and this is consistent with the performance characteristics reported in several recent studies that have used direct inoculation of nasal swab specimens. The excellent inhibition of resistant Gram-negative organisms as well as nasopharyngeal flora allowed for a clear visualization of highly specific mauve colonies. However, mauve colonies on CSA/CSA-MRSA should be confirmed directly from plates using the Staphaurex slide test or the tube coagulase test before the presumptive reporting of $S$. aureus/MRSA.

\section{ACKNOWLEDGEMENTS}

We thank the technical staff of the Clinical Microbiology Laboratory for their assistance in this study. This work was presented in part at the Interscience Conference on Antimicrobial Agents and Chemotherapy, Washington, DC, December 182005 (presentation number D-1724, published by the American Society for Microbiology).

\section{REFERENCES}

Clinical and Laboratory Standards Institute (2005). Performance Standards for Antimicrobial Susceptibility Testing; Fifteenth Informational Supplement. Wayne, PA: Clinical and Laboratory Standards Institute.

Diederen, B., van Duijn, I., van Belkum, A., Willemse, P., van Keulen, P. \& Kluytmans, J. (2005). Performance of CHROMagar MRSA medium for detection of methicillin-resistant Staphylococcus aureus. J Clin Microbiol 43, 1925-1927.

Flayhart, D., Hindler, J. F., Bruckner, D. A., Hall, G., Shrestha, R. K., Vogel, S. A., Richter, S. S., Howard, W., Walther, R. \& Carroll, K. C. (2005). Multicenter evaluation of BBL CHROMagar MRSA medium for direct detection of methicillin-resistant Staphylococcus aureus from surveillance cultures of the anterior nares. J Clin Microbiol 43, 5536-5540.

Francois, P., Pittet, D., Bento, M., Pepey, B., Vaudaux, P., Lew, D. \& Schrenzel, J. (2003). Rapid detection of methicillin-resistant Staphylococcus aureus directly from sterile or nonsterile clinical samples by a new molecular assay. J Clin Microbiol 41, 254-260.

Gaillot, O., Wetsch, M., Mortineau, N. \& Berche, P. (2000). Evaluation of CHROMagar Staph aureus, a new chromogenic medium for isolation and presumptive identification of Staphylococcus aureus from human clinical specimens. J Clin Microbiol 38, 1587-1591.

Muto, C. A., Jernigan, J. A., Ostrowsky, B. E., Richet, H. M., Jarvis, W. R., Boyce, J. M. \& Farr, B. M. (2003). SHEA Guideline for preventing nosocomial transmission of multi-resistant strains of Staphylococcus aureus and Enterococcus. Infect Control Hosp Epidemiol 24, 362-386.

Perry, J. D., Renninson, C., Butterworth, L. A., Hopley, A. L. \& Gould, F. K. (2003). Evaluation of S. aureus ID, a new chromogenic agar 
medium for detection of Staphylococcus aureus. J Clin Microbiol 41, 5695-5698.

Perry, J. D., Davies, A., Butterworth, L. A., Hopley, A. L., Nicholson, A. \& Gould, F. K. (2004). Development and evaluation of a chromogenic agar medium for methicillin-resistant Staphylococcus aureus. J Clin Microbiol 42, 4519-4523.

Rubin, R. J., Harrington, C. A., Poon, A., Dietrich, K., Greene, J. A. \& Moiduddin, A. (1999). The economic impact of Staphylococcus aureus infection in New York City hospitals. Emerg Infect Dis 5, 9-17.

Stoakes, L., Reyes, R., Daniel, J., Lennox, G., John, M. A., Lannigan, R. \& Hussain, Z. (2006). Prospective comparison of a new chromogenic medium, MRSASelect, to CHROMagar MRSA and mannitol-salt medium supplemented with oxacillin or cefoxitin for detection of methicillin-resistant Staphylococcus aureus. J Clin Microbiol 44, 637-639.

Swenson, J. M., Tenover, F. C. \& The Cefoxitin Disk Study Group (2005). Results of disk diffusion testing with cefoxitin correlate with presence of mecA in Staphylococcus spp. J Clin Microbiol 43, 3818-3823.

Warren, D. K., Liao, R. S., Merz, L. R., Eveland, M. \& Dunne, W. M. (2004). Detection of methicillin-resistant Staphylococcus aureus directly from nasal swab specimens by a real-time PCR assay. J Clin Microbiol 42, 5578-5581.

Wernitz, M. H., Keck, S., Swidsinski, S., Schulz, S. \& Veit, S. K. (2005). Cost analysis of a hospital-wide selective screening program for methicillin-resistant Staphylococcus aureus (MRSA) carriers in the context of diagnosis related (DRG) payment. Clin Microbiol Infect 11, 466-471. 